\title{
Adaptación lingüística, estructura factorial y fiabilidad del Inventario de Perspectiva Temporal de Zimbardo para Buenos Aires
}

\author{
Linguistic adaptation, factorial structure and reliability of Zimbardo Time \\ Perspective Inventory for Buenos Aires
}

María Elena Brenlla ${ }^{1}$, Joaquín Zapater ${ }^{2}$ y Guadalupe Germano ${ }^{3}$

${ }^{1}$ Doctora en Psicología. Investigadora en el Centro de Investigaciones en Psicología y Psicopedagogía (CIPP) de la Universidad Católica Argentina (UCA). Profesora titular de Técnicas de Evaluación Psicológica I, Facultad de Psicología y Psicopedagogía, Universidad Católica Argentina. E-mail: bren@uca.edu.ar

${ }^{2}$ Licenciado en Psicología. Asistente de investigación en el Centro de Investigaciones en Psicología y Psicopedagogía (CIPP) de la Universidad Católica Argentina.

${ }^{3}$ Licenciada en Psicología. Becaria doctoral del Consejo Nacional de Investigaciones Científicas y Técnicas (CONICET) en el Centro de Investigaciones en Psicología y Psicopedagogía (CIPP). Profesora asistente de Metodología de la Investigación I, Facultad de Psicología y Psicopedagogía, Universidad Católica Argentina. E-mail: guadalupe_germano@uca.edu.ar

Centro de Investigaciones en Psicología y Psicopedagogía (CIPP), Universidad Católica Argentina. Ciudad Autónoma de Buenos Aires, Argentina.

\section{Resumen}

La perspectiva temporal es un término psicológico que refiere al modo en que las personas ordenan sus experiencias en categorías temporales. Se asocia con la salud mental, las conductas de riesgo y los proyectos a futuro. El Inventario de Perspectiva Temporal de Zimbardo (ZTPI; Zimbardo y Boyd, 1999) es un cuestionario autoadministrable que evalúa la perspectiva temporal. En este artículo se informan los resultados del estudio psicométrico del ZTPI, realizado en Buenos Aires, sobre la base de la adaptación española del inventario. En este sentido, un propósito fundamental fue analizar si la adapta- ción española del ZTPI es igualmente válida para su uso en Argentina. Para ello, se llevó a cabo una adaptación lingüística del instrumento que redundó en cambios en la redacción y contenido en la presente versión para adecuarlas a las particularidades del habla en Argentina. Con esta versión, se realizó un estudio piloto con una muestra de 323 personas adultas cuyos datos permitieron el análisis básico de sus propiedades psicométricas. Las evidencias de validez interna corroboran la estructura de cinco componentes del estudio original. La fiabilidad de los ítems en cada factor fue satisfactoria (valores $\alpha$ entre .59 y .78). Los resultados de validez externa muestran asociaciones significativas 
entre pasado negativo y malestar psicológico $(r=.43 ; p<.005)$, presente fatalista y locus de control $(r=.45 ; p<.005)$, presente hedonista y autoeficacia generalizada $(r=.31$; $p<.005)$. Respecto a la variabilidad obtenida en las puntuaciones del ZTPI se sugiere que, en parte, se debe al nivel educativo $y$, secundariamente, al sexo. Conforme aumenta la edad, el patrón de orientación al presente hedonista decrece. Lo contrario sucede con el predominio de la orientación futura.

Palabras clave: Perspectiva del tiempo; Inventario; Adaptación; Fiabilidad; Validez.

\section{Abstract}

Time perspective is a psychological term that refers to the process by which a person sorts out personal experiences into temporal categories. It is associated with mental health, risk behaviors, cognitive and emotional empathy, and future goals. The Zimbardo Time Perspective Inventory (ZTPI) from Zimbardo \& Boyd (1999) is a self-administered questionnaire consisting in 56 items with 5 answer options. It examines time perspective in a quantifiable way. The questionnaire was translated and adapted to many languages and cultures, most of which reported satisfactory psychometric evidences. The Spanish version of the ZTPI was first made in Spain by Diaz-Morales (2006). Based on this version, two other Spanish speaking countries, Chile and Mexico, made their own version of the questionnaire. In Argentina, there is a version of the ZTPI valid only for teenagers. In the present work the original ZTPI was translated by 3 experts and a linguistic adaptation was done in order to adequate the items to Argentinean speech. A pilot test using the Diaz-Morales version was carried out. Based on the independent and blinded translations, and on the results of the pilot test, an analysis was made. Many items of the Spanish versions were kept, but 34 of the 56 items were changed so as to be compressible for Argen- tinians. This work resulted in an Argentinian version of the ZTPI, specially done for that culture. Using this version, a pilot study was done with a sample of 323 adults from Buenos Aires. The analysis shows that the reliability of items was satisfactory (values between $a$ $=.59$ to $a=.78$ ). Internal validity evidences confirm the 5 factors structure shown by the authors. External validity was analyzed showing significant associations among negative past and psychological distress $(r=.43$; $p<.005$ ), present fatalistic and locus of control $(r=.45 ; p<.005)$ and present hedonistic and Self-efficacy $(r=.31 ; p<.005)$. To study the error variance due to passing of time, test-retest was carried out, showing high and positive correlations. This means that scores of time perspective show time stability. In conclusion, the results of the psychometric analysis are satisfactory. This indicates that the ZTPI is a reliable and valid instrument that can be used to evaluate time perspective in adults from Buenos Aires. An analysis of the variability of ZTPI punctuations was done. It suggests that they are due to two main reasons: educational level and sex. Regarding educational level, significant variations were shown in four of the five factors. The only factor that showed no difference was the positive past. Considering sex, significative differences were shown in four of the five factors of the test, in which medias were higher in women than in men. No differences were shown in hedonistic present factor. With respect to the age, present hedonistic orientation decrease as age grows. On the other hand, as age grows future orientation grows too. These three variables (educational level, sex and age) explain the variability, but it is a partial significance. This implies that time orientation can be understood as a personality characteristic. Consequently, the different punctuations people get in the ZTPI are due to individual differences. Suggestions are made in order to make a definitive adaptation of the ZTPI for Argentina. The most important are increasing the sample with people from other regions of the country and increase 
the sample to make test-retest. Finally, future research could be oriented to make a short version of the ZTPI and use the questionnaire for evaluation in clinical samples to understand how time perspective works in psychopathologies and then use time orientation as a valid variable for diagnosis and treatment.

Keywords: Time perspective; Inventory; Adaptation; Reliability; Validity.

\section{Introducción}

El estudio del tiempo y su relación con la conciencia humana ha sido, a lo largo de la historia de la filosofía y la psicología, un tema recurrente (Roeckelein, 2000). James (1945), padre de la psicología norteamericana, puso de relieve la importancia de la percepción del tiempo en psicología al dedicarle un capítulo entero en su tratado Principles of Psychology. Esta recurrencia parece deberse a que tanto el tiempo como el espacio son coordenadas de las que se vale el hombre para referirse a fenómenos que ocurren en el mundo, y a la idea de que éste está estructurado en parámetros espacio-temporales. Por su parte, Piaget (1970) supone que la adquisición de las nociones temporales estaría íntimamente relacionada con la adquisición y dominio del lenguaje. Según el autor, el niño comienza viviendo en el presente, por lo que, entre los 18 y los 20 meses, las escasas palabras temporales de que dispone están referidas al presente y solo posteriormente se adquieren palabras relacionadas con el futuro, y por último con el pasado.

Respecto a las investigaciones en psicología, a mitad del siglo XX se dio un aumento inusitado de estudios sobre los fenómenos temporales. Entre los más relevantes se encuentran aquellos que evalúan la predominancia de uno de los tres dominios temporales -pasado, presente, futuro- en la determinación de la conducta. Así, Nuttin (1985) enfatizó la perspectiva futura mientras que los conductistas se enfocaron en el pasado debido a su interés en explicar la conducta, en virtud de la interrelación de los contextos ambientales con los condicionamientos sucedidos (Skinner, 1985). Entre los intentos más relevantes de operacionalizar el fenómeno del tiempo psicológico, se encuentra el Método de Inducción Motivacional (MIM; Nuttin y Lens, 1980) que explora metas y temores frente al futuro y los ubica en una escala temporal, la Escala de Ansiedad Futura de Zaleski (1996) y la Escala de Consideración de las Consecuencias Futuras (Strathman, Gleicher, Boninger y Edwards, 1994) que está adaptada al español (Vásquez Echeverría, Martín, Ortuño, Esteves, y Joireman, 2017). Asimismo, se encuentra la escala Long Term Personal Direction Scale (LTPD) (Wessmann, 1973), que fue y sigue siendo muy utilizada en el campo de la orientación vocacional u orientación para la carrera, y los estudios de Janeiro (2012), quien propone un instrumento para evaluar tres zonas temporales en estudiantes de colegio secundario de Portugal. La mayoría de estos cuestionarios -salvo el de Janeiro- tienen en común centrarse en una sola dimensión del tiempo psicológico y, al ser unidimensionales, no logran captar cómo se relacionan entre sí las diferentes dimensiones.

Zimbardo y Boyd (1999) fueron pioneros en introducir la evaluación integral de la orientación temporal a través del diseño del Inventario de Perspectiva Temporal de Zimbardo (ZTPI). La orientación temporal es una dimensión elemental y específica del constructo de tiempo psicológico, y emerge del proceso cognitivo que divide la experiencia humana en dimensiones temporales de pasado, presente y futuro. Es necesario mencionar que, en cuanto al estudio de la percepción del tiempo, pueden diferenciarse dos conceptos centrales: la perspectiva temporal y la orientación temporal (Holman y Silver, 1998). La perspectiva temporal es la distancia cognitiva entre las metas y las experiencias vividas (Nuttin, 1985), mientras que la orientación temporal es la tendencia que tiene el sujeto a estar ubicado en un marco 
temporal específico: pasado, presente o futuro (Lewin, 1978; Zimbardo y Boyd, 1999). Estos marcos ayudan a ordenar la experiencia y darle orden, coherencia y sentido a nuestras vidas (Zimbardo y Boyd, 1999). Si bien Zimbardo y Boyd (1999) utilizan el término perspectiva, no obstante, y acorde a la diferenciación indicada, el ZTPI evalúa, en realidad, orientación temporal. Cabe destacar que esta puntualización fue realizada por Díaz Morales (2006) en su adaptación española del test.

Como se anticipó, en 1999 Zimbardo y Boyd diseñaron y publicaron el ZTPI cuyo objetivo era evaluar, de manera cuantificable, la orientación temporal. Se trata de un cuestionario autoadministrable que consta de 56 ítems con 5 opciones de respuesta tipo Likert. Los ítems versan acerca de las creencias, preferencias y valores con respecto a la experiencia ubicada dentro de un marco temporal. La técnica proporciona una manera de medir las dimensiones temporales de un individuo y brinda como resultado un perfil de orientación temporal.

Los autores plantean que estas orientaciones temporales ejercen una influencia dinámica en los juicios, decisiones y acciones importantes que realizan las personas. Cuando un sujeto sobreenfatiza una dimensión particular se produce un sesgo. En caso de que el sesgo esté puesto en algunas de las orientaciones pasadas, la influencia dominante para tomar una decisión vendrá del pasado, a través del recuerdo de alguna situación análoga y los costos y beneficios asociados a aquella situación. A su vez, este recuerdo puede tener diferentes valencias afectivas que pueden alterar el recuerdo del pasado. En cambio, cuando el sesgo está puesto en el futuro, la influencia dominante provendrá de una expectativa o anticipación que construye el sujeto y que funciona como una extensión del presente hacia el futuro. En caso de que el sesgo esté puesto en el presente, la persona se encuentra imposibilitada de trascender el estímulo inmediato, dificultándose la capacidad de sostener la demora de la gratificación. En estos casos, la influencia dominante suele provenir de fuentes sensoriales, biológicas y sociales asociadas a las características salientes del estímulo. El sujeto estaría sesgado al estar más orientado al pasado, futuro o presente. Estos sesgos pueden incluir el uso habitual y excesivo de una orientación, así como también el uso escaso de otra. Cuando este sesgo se vuelve crónico se transforma en un estilo de funcionamiento característico y predecible de cómo el sujeto va a actuar (Zimbardo y Boyd, 1999).

Existe también la orientación temporal balanceada, la cual permitiría al sujeto poder cambiar de manera flexible el marco temporal con el que se está manejando en ese momento. La necesidad de cambiar estaría determinada por demandas situacionales, preferencias personales y/o sociales, entre otras. El comportamiento de las personas cuya orientación temporal es balanceada estaría determinado por las experiencias pasadas, los deseos del presente y las consecuencias futuras (Zimbardo y Boyd, 1999).

Entre los antecedentes teóricos de la técnica, cabe mencionar en primer lugar a Lewin (1978), -quien planteó que tanto el pasado como el futuro existen de manera simultánea en un mismo campo psicológico en un momento determinado, y que fue pionero en establecer la noción de perspectiva temporal-, así como a Nuttin $(1964,1985)$ quien expresa que el pasado y el futuro influyen en cómo las personas se desenvuelven en el presente. De esta misma manera, el trabajo realizado por Zimbardo y Boyd (1999) se extiende sobre la idea lewineana y describen la perspectiva temporal como un proceso fundamental para el funcionamiento tanto individual como social. Ellos entienden la perspectiva temporal como una variable desde una concepción situacionalista, pero que a la vez, parece funcionar como un proceso relativamente estable a nivel individual.

Fraisse (1984), cuyos trabajos se enmarcan en el situacionalismo, plantea que el futuro próximo se caracteriza por ser una proyección 
del pasado y que debe diferenciarse del futuro prospectivo, que refiere a aquellas metas no alcanzables en el presente. El autor introduce el término horizonte temporal para designar la distancia prospectiva de las proyecciones que hace un sujeto. Esta es una variable que depende de las experiencias de los padres, la edad y situaciones coyunturales asociadas a cómo se vivan las satisfacciones del presente (Fraisse, 1984). Este horizonte temporal podría asimilarse a lo que Zimbardo (1999) denomina orientación temporal futura, $\mathrm{y}$ conjetura que cuanto más plana es la distancia prospectiva del horizonte temporal, habrá una tendencia más marcada a estar orientado temporalmente al presente, tanto hedonista como fatalista.

Zimbardo y Boyd (2008), basándose en sus investigaciones, consideran que existen tres paradojas básicas que ayudan a comprender el alcance y lo complejo del fenómeno del tiempo. La primera plantea que el tiempo ejerce una de las influencias más poderosas en cómo uno piensa, actúa y siente, pero que, sin embargo, esta gran influencia del tiempo queda, la mayoría de las veces, completamente desapercibida. La segunda paradoja se basa en que todas las orientaciones temporales se encuentran asociadas a numerosos beneficios, pero que estar excesivamente orientado hacia alguna dimensión temporal está asociado con mayor cantidad de costos o dificultades que de beneficios. La tercera y última paradoja es que, pese a que las orientaciones temporales se van forjando en base a la experiencia individual, puede funcionar como un fenómeno colectivo y así influir en el devenir de una sociedad o un país.

Finalmente, cabe mencionar que la orientación temporal ha sido asociada a una cantidad de condiciones psicológicas y psicosociales como las conductas de riesgo, la tendencia a las adicciones y la conducta temeraria en la conducción de automóviles (Keough, Zimbardo y Boyd, 1999; Zimbardo y Boyd, 1999), principalmente porque es un proceso cognitivo, en el que interviene la experiencia del pasado y las expectativas del futuro, que determina cómo se actúa en el presente (Zimbardo y Boyd, 1999). Oyanadel y BuelaCasal (2010) pusieron de manifiesto la relación entre la orientación temporal y el nivel de salud física y mental. Demostraron que determinados perfiles temporales, como la orientación temporal balanceada, correlacionan positivamente con la salud, mientras que otros, pasado negativo por ejemplo, lo hacen de manera negativa.

Ahora bien, ¿cómo llegan los autores a desarrollar esta técnica? La idea de desarrollar un cuestionario que midiese la perspectiva temporal comenzó a partir de lo que Zimbardo observó en el experimento de la cárcel de Stanford (Zimbardo, Haney, Banks y Jaffe, 1973). La situación particular de encarcelamiento tenía el poder de alterar las orientaciones temporales de varios de sus participantes. Los estudiantes que participaron, estando orientados al futuro principalmente, pasaron a ser prisioneros completamente inmersos en el presente, sin ningún interés por su pasado ni por su futuro. A partir de esta experiencia se inicia el desarrollo del ZTPI, cuya versión final (Zimbardo y Boyd, 1999) es el corolario de un trabajo exhaustivo que ha durado varios años. El refinamiento de la técnica se hizo de manera empírica con validaciones experimentales y a través de varios análisis factoriales para identificar los ítems que pudieran caracterizar las diferentes dimensiones temporales.

En relación con las propiedades psicométricas, Zimbardo y Boyd (1999) informan que las puntuaciones del ZTPI, sometidas a análisis factorial, se agrupan en cinco factores que explican el $36 \%$ de la varianza. Los cinco factores identificados fueron: pasado negativo -que supone una actitud negativa y pesimista del pasado-, presente hedonista -que implica una actitud de disfrute del momento actual sin evaluar las consecuencias-, futuro -entendido como la tendencia a planificar metas a largo plazo-, pasado positivo -que refleja una actitud feliz y nostálgica con respecto al pasado-, y presente fatalista, que supone una 
actitud de indefensión con respecto a la vida en general (Zimbardo y Boyd, 1999).

En términos de validez convergente y divergente, los autores informan que la naturaleza de la variable y sus factores estarían asociados a otros constructos. El factor pasado negativo correlacionó positivamente con agresión, depresión, ansiedad y correlacionó negativamente con estabilidad emocional y autoestima. El factor presente hedonista se asoció con búsqueda de novedad y sensaciones nuevas, y negativamente con preferencia por la consistencia. El factor futuro correlacionó positivamente con estabilidad emocional, consideración de las consecuencias futuras, preferencia por la consistencia y recompensa, y correlacionó negativamente con búsqueda de novedad. El factor presente fatalista correlacionó positivamente con agresión, depresión $\mathrm{y}$ ansiedad, mientras que correlacionó negativamente con consideración de consecuencias futuras (Zimbardo y Boyd, 1999).

Además de estos hallazgos que relacionan la salud mental con la orientación temporal, en este estudio se conjeturan asociaciones específicas con dos percepciones sociocognitivas: la de autoeficacia y la de locus de control. Por eso, para evaluar evidencias de validez convergente, se utilizaron escalas que miden estos constructos. En particular, se espera que la Escala de Autoeficacia Generalizada (Schwarzer y Jerusalem, 1995) correlacione positivamente con el factor presente hedonista, ya que implica la capacidad de ser asertivo y tener confianza en las propias habilidades para la adaptación. Como opuesto a esto, se esperaría que la Escala de Locus de Control (Rotter, 1966) correlacione positivamente con presente fatalista y con pasado negativo. La Escala de Locus de Control evalúa qué estilo de locus de control (LOC) tiene el sujeto pudiendo ser éste externo o interno. El LOC refiere a una expectativa generalizada de control sobre los esfuerzos. Aquellas personas que tienen LOC interno poseerían la expectativa de que los resultados que obtienen son contingentes o causados por sus propias conductas o características personales. Los sujetos con LOC externo, por su parte, percibirían que las consecuencias de sus conductas no dependen de su propio control, sino a factores ajenos como la suerte, el destino o la participación de otras personas (Rotter, 1966).

El ZTPI es un inventario que ha sido adaptado a numerosos países. La mayoría de las adaptaciones han mostrado evidencias psicométricas satisfactorias (Apostolidis, Fieulaine, Simonin y Rolland, 2006; Díaz Morales, 2006; Kolesovs, 2009; Milfont, Andrade, Belo y Pesoa, 2008; Sircova et al., 2014). Para una revisión exhaustiva de las adaptaciones del ZTPI en el mundo consúltese el artículo de Galarraga y Stover (2016).

Díaz Morales (2006) fue quien adaptó por primera vez el inventario al idioma español. El análisis de la muestra española arrojó una estructura en cinco factores bastante similar a la obtenida en muestras norteamericanas. La composición de los factores pasado positivo $\mathrm{y}$ presente fatalista posee algunos elementos que no coinciden con la versión original y esto podría deberse a diferencias culturales. Al evaluar diferencias por edad y sexo, el autor informa que los grupos de mayor edad mostraron mayor orientación al futuro y menor al presente hedonista, las mujeres presentaron mayor orientación al pasado negativo, pasado positivo y presente fatalista que los hombres. En relación a las limitaciones, en el artículo se indica que una de las más relevantes es no haber analizado la validez externa del ZTPI en su relación con otras variables psicológicas criterio.

Otras adaptaciones al español son las realizadas en Chile por Oyanadel, BuelaCasal y Pérez-Fortis (2014) y en México por Corral-Verdugo, Fraijo-Sing y Pinheiro (2006). En Argentina, se realizó una adaptación del ZTPI para adolescentes (Galarraga y Stover, 2016), específicamente, con estudiantes de nivel medio de Buenos Aires que difiere del rango etario considerado en la versión española y justifica, en tal sentido, 
el estudio que en este artículo se informa. En la adaptación para adolescentes se aisló una estructura temporal de cuatro dimensiones, en lugar de las cinco propuestas por Zimbardo y Boyd (1999), que dio lugar a una reducción de la cantidad de ítems. Las autoras proponen que estas diferencias se deben fundamentalmente a cambios culturales y a la diferencia de la edad de los evaluados.

Ahora bien, sabiendo que existen diferentes versiones válidas en el idioma español cabe preguntarse ¿por qué es necesario hacer una adaptación argentina? Esto nos lleva a un tema de interés en la investigación psicométrica: el de la equivalencia lingüística y cultural de un test. En concreto, ¿es suficiente que haya una versión de un test en español realizada en un país particular para que esta versión sea igualmente válida en los demás países que comparten la lengua?

La respuesta es no. En el caso concreto de la comparación del uso del español en Argentina y en España, existen ciertos sustantivos comunes en España - por ejemplo, falda- que son muy poco frecuentes en Argentina y, lo que es más complejo aún, ciertos adjetivos que aluden a emociones - como enfadado- que no son conocidos en Argentina, o ciertos verbos cuya acepción más frecuente es completamente diferente en España y Argentina como en el caso del verbo tomar-. A la vez, ciertas expresiones como "me hace ilusión" son desconocidas en Argentina, pero de uso corriente en España. Marín (1986), en un artículo pionero por su temática, alertó acerca de los problemas devenidos de estas variaciones lingüísticas, sutiles u ostensibles, de los hablantes de un mismo idioma. La cuestión fundamental de ello reside en el impacto que tiene en la validez de contenido de la prueba y consecuentemente, en la validez de constructo y en el error de medición.

Poortinga (1989) sugiere que la comparación entre culturas requiere equivalencia de datos y ésta involucra dos conceptos: equivalencia de constructo y equivalencia de medición. En el caso de la adaptación de instru- mentos de medida, implica una revisión de la adaptación lingüística y de sus propiedades psicométricas. En consecuencia, no puede considerarse que la adaptación a un idioma realizada en un país deba adoptarse sin más a otro país, aunque el idioma sea el mismo.

Por lo antedicho, el propósito esencial del trabajo que se informa es conocer si la adaptación española del ZTPI es apropiada para su uso en Argentina y brindar información acerca de las propiedades psicométricas del ZTPI en Buenos Aires. El objetivo es proporcionar una versión fiable y válida del inventario a fin de poder evaluar población clínica en futuras investigaciones y diseñar nuevas estrategias de prevención y de tratamiento que incluyan abordajes de la temporalidad. Cabe destacar que se contactó a los autores del ZTPI y se cuenta con su aprobación para la ejecución de la adaptación (N. Feulaine, comunicación personal, 13 de septiembre de 2014).

\section{Método}

\section{Diseño y muestra}

Para estudiar los objetivos se apeló a un tipo de estudio descriptivo transversal con diseño correlacional. El muestreo fue no probabilístico, con una muestra de 323 participantes, adultos, residentes en la Ciudad Autónoma de Buenos Aires y en el Gran Buenos Aires, sin antecedentes neurológicos y psiquiátricos, ni retraso mental. Las personas tenían edades comprendidas entre los 18 y 32 años $(M=34.94$; $D E=13.417)$, de las cuales el $56 \%$ eran mujeres. Para describir la variable edad se dividió a los participantes en tres grupos: muy jóvenes, de 18 a 22 años (27.9 \%); jóvenes, de 23 a 45 años (42.7\%); adultos, de 46 a 62 años $(29.4 \%)$. Esta clasificación está basada en la descripción de las etapas de desarrollo psicosocial que Bordignon (2005) propone en base a las descriptas por Erikson. Cada participante indicó también su máximo nivel de estudios alcanzados, observándose que el $30 \%$ tenía estudios superiores, 
el 53.6\% había cursado el secundario completo y el $16.4 \%$ el secundario incompleto.

\section{Procedimiento}

En primer lugar, se solicitó autorización a los autores del ZTPI para la realización de este estudio (N. Fieulaine, comunicación personal, 13 de septiembre de 2014). Dado que el ZTPI se originó en Estados Unidos en inglés, para adaptar la prueba al medio local se realizaron tres traducciones lingüísticas independientes y a ciegas. Éstas fueron comparadas con la versión en español de la escala desarrollada por Díaz Morales (2006) y con la versión original en inglés. A la vez, 82 personas contestaron la versión española con la indicación de consignar si los ítems les parecían claros, tanto sintáctica como semánticamente (Brenlla, Serna, Londra, Rodríguez y Zapater, 2012). Luego, se llevó a cabo un estudio de juicio de expertos para evaluar la validez de contenido del ZTPI en términos de su relevancia, coherencia, claridad y suficiencia para evaluar las 5 orientaciones temporales. A partir del análisis de los tres jueces y de las opiniones de los entrevistados se pasó de las versiones experimentales a la definitiva que se utilizó en esta investigación.

El procedimiento de la recolección de datos fue de participación voluntaria con el uso de consentimiento informado. A partir del trabajo de campo $(n=323)$ se realizaron los análisis estadísticos correspondientes.

\section{Instrumentos}

1.- Inventario de Perspectiva Temporal de Zimbardo ZTPI (Zimbardo y Boyd, 1999). Se utilizó para evaluar la orientación temporal. La orientación temporal se define operacionalmente como la experiencia asociada a creencias, preferencias $\mathrm{y}$ valores ubicados dentro de una dimensión temporal. Esto remitiría a la orientación temporal autopercibida del sujeto. El ZTPI es un cuestionario autoadministrable que consta de 56 ítems con 5 opciones de respuesta tipo Likert, que se puntúan con valores de 1 a 5 , de la siguiente manera: 1 : Completamente falso, 2: Bastante falso, 3: Neutral, 4: Bastante verdadero, 5: Completamente verdadero. El inventario cuenta con 5 ítems que deben ser recodificados (ítems 9, 24, 25, 41 y 56). Las respuestas de estos ítems recodificados se puntúan con valores de 5 a 1 .

2.- Escala de Locus de Control (Rotter, 1966). Es un autoinforme que consta de 29 ítems con opciones de respuesta de elección forzada que marcan diferentes percepciones de control: externas (creencias de que la propia conducta se haya condicionada por el destino u otros poderosos) o internas (creencias de que el individuo es responsable de su conducta y que ésta influye de manera positiva en su entorno). Se asigna una puntuación cada vez que se refiere al control externo, la cual puede oscilar entre 0 y 23 puntos dado que 6 ítems funcionan como distractores. Es decir que el aumento de la puntuación indicaría predominio de creencias de control externo. Se trabajó con una adaptación de Brenlla, Vázquez y Aranguren (2008). En la adaptación argentina, los coeficientes de fiabilidad son aceptables $(\alpha=.64)$ y se comprobaron correlaciones con escalas que miden constructos afines, como el afrontamiento centrado en la emoción $(r=.31)$ y el riesgo de malestar psicológico $(r=.40)$.

3.- Escala de Autoeficacia Generalizada (Schwarzer y Jerusalem, 1995). La autoeficacia general es el constructo global que se refiere a la creencia estable de una persona acerca de su capacidad para manejar diversos tipos de estresores de la vida cotidiana. La escala se compone de 10 ítems con 4 opciones de respuesta tipo Likert. Las puntuaciones totales pueden variar entre 10 y 40 puntos. Las más altas son indicadores de una mayor creencia de autoeficacia general. Se trabajó con la adaptación argentina (Brenlla, Aranguren, 
Rossaro y Vázquez, 2010) que presentó evidencias de fiabilidad muy satisfactorias $(\alpha=.76)$ y de validez externa con correlaciones significativas con constructos independientes tales como actividad $(r=.39)$, locus de control externo $(r=-.29)$ y estrategias de afrontamiento al estrés de planeamiento $(r=.45)$.

4.- Escala de Malestar Psicológico de Kessler K10 (Kessler y Mrozeck, 1994). Evalúa el riesgo de presentar malestar psicológico inespecífico (síntomas de ansiedad y/o depresión) en el último mes. Está compuesta de 10 ítems con un escalamiento tipo Likert de 5 respuestas. El rango de puntuaciones oscila entre los 10 y 50 puntos. Se trabajó con la adaptación argentina de Brenlla y Aranguren (2010), cuyas evidencias de fiabilidad $(\alpha=.88)$, de estabilidad $(r=.36)$ $\mathrm{y}$ de validez con constructos independientes de depresión $(r=.89)$ y ansiedad $(r=.84)$ fueron muy satisfactorias.

\section{Procesamiento de los datos}

Acorde a los objetivos específicos, primero se analizó la pertinencia lingüística de la versión de Díaz Morales (2006) para su uso en la Argentina y las evidencias de validez de contenido, a través del porcentaje de acuerdo entre evaluadores. Luego se realizó un análisis factorial exploratorio a fin de conocer si la estructura se asemejaba a la original, un análisis de la consistencia interna -mediante el uso del coeficiente Alpha de Cronbach- y un análisis de correlaciones para estudiar las evidencias de validez externa. La estabilidad de las puntuaciones se analizó a través del método de test-retest y se calculó sobre la base de un estudio de correlación entre dos conjuntos de puntuaciones.

\section{Resultados}

En relación con la adaptación lingüística, se analizaron los resultados del análisis de la adaptación original del ZTPI (Zimbardo y Boyd, 1999) realizado por los tres jueces bilingües independientes y por las respuestas de 82 personas que contestaron la versión española (Díaz Morales, 2006), con la indicación de consignar si los ítems les parecían claros, tanto sintáctica como semánticamente (Brenlla et al., 2012). En total, se varió la redacción de 34 ítems de la versión española. Algunos ejemplos de los ítems que fueron modificados para la versión argentina del ZTPI son: el ítem 16 "Las experiencias dolorosas del pasado permanecen en mi memoria", por "Revivo constantemente experiencias dolorosas del pasado"; el ítem 22 "He tenido mi ración de abuso y rechazo en el pasado", por "Tuve suficiente con mi cuota de maltrato y rechazo en el pasado"; el ítem 30 en el cual se cambió el término "costes" por "costos"; y el ítem 41 "Me encuentro "fuera de onda» cuando los miembros de mi familia hablan de cómo solían ser las cosas", por "Me encuentro «fuera de sintonía» cuando mi familia habla de cómo solían ser las cosas".

A partir del análisis de los tres jueces y de las opiniones de los entrevistados, se pasó de las versiones experimentales a la definitiva que se utilizó en esta investigación. Luego, se llevó a cabo un estudio de juicio de expertos para evaluar la validez de contenido del ZTPI en términos de su relevancia, coherencia, claridad y suficiencia. Para determinar el nivel de acuerdo entre examinadores se utilizó el porcentaje de acuerdo entre examinadores. Fueron dos examinadoras, ambas psicólogas con dedicación a la clínica; una de ellas también se dedicaba a la investigación. Los vectores que se analizaron fueron los de claridad, coherencia y relevancia. La claridad busca explorar si el ítem es de fácil comprensión, y si su sintáctica y semántica son adecuadas. La coherencia estudia si el ítem tiene relación lógica con la dimensión que pretende medir. Por último, la relevancia explora la importancia del ítem, es decir, si debe ser incluido para la medición de la dimensión (Escobar-Pérez y Cuervo-Martínez, 2008). El resultado en las tres dimensiones estudiadas fue significativo, variando entre $71 \%$ y $59 \%$. 
La dimensión de relevancia demostró tener el mayor grado de concordancia (71\%), seguido por la coherencia $(67 \%)$ y por último, la claridad (59\%).

Es importante aclarar que la recodificación de los ítems obedeció a que se encontraban redactados de manera inversa a lo que el factor pretende explorar. Un claro ejemplo sería el ítem 24 que dice "Intento tomarme el día como viene, sin intentar planificarlo" que carga en el factor futuro. Ello explica por qué los jueces indicaron a estos ítems como poco relevantes, coherentes o claros. Una vez que les fue aclarado que estos ítems estaban redactados de manera inversa, variaron su juicio positivamente.

Las evidencias de validez estructural se analizaron con el mismo procedimiento que los autores del inventario. De acuerdo con los criterios de Martínez Arias (1995) respecto de la cantidad de observaciones necesarias para realizar análisis factorial, la cantidad de casos incluidos en este estudio $(n=320)$ resulta exigua, pero suficiente para un análisis básico. En primer lugar se realizó una inspección visual del gráfico de sedimentación que mostró que cinco factores eran viables. El índice de adecuación muestral Kaiser-Meyer-Olkin (KMO) fue de .75, que señala una matriz de correlaciones aceptable para el uso del método de componentes principales. El análisis brindó cinco factores que explicaron el $34.46 \%$ de la varianza $(10.01 \%, 7.2 \%, 6.1$ $\%, 5.94 \%, 5.22 \%$ ). La mayoría de los ítems del cuestionario tuvieron una relación significativa con el factor en el que se esperó que cargaran. Todos los ítems -con excepción del ítem 48, que tuvo una carga de .28-, presentaron cargas factoriales superiores a .35. A su vez, las evidencias de consistencia interna de los cinco factores fueron satisfactorias.

El primer factor fue identificado como pasado negativo y su coeficiente de fiabilidad es de $\alpha=.78$. En relación con la prueba original, hay una concordancia de 9 ítems sobre un total de 10. El segundo factor fue identificado como presente hedonista y su coeficiente de fiabilidad es de $\alpha=.78$. En relación con la prueba original hay una concordancia de 8 ítems de 15. El tercer factor fue identificado como futuro y su coeficiente de fiabilidad es de $\alpha=.65$. En relación con la prueba original hay una concordancia de 7 sobre un total de 13 ítems. El cuarto factor fue identificado como presente fatalista y su coeficiente de fiabilidad es de $\alpha=.66$. En relación con la prueba original hay una concordancia de 2 sobre un total de 9 ítems. El quinto factor fue identificado como pasado positivo y su coeficiente de fiabilidad es de $\alpha=.59$. En relación con la prueba original hay una concordancia de 7 de 9 ítems. En la discusión se explicará la diferencia en las concordancias.

En términos de validez externa, el factor pasado negativo correlacionó positivamente con la Escala de Locus de Control ( $r=.31$; $p<.05)$ y con la Escala de Malestar Psicológico $(r=.43 ; p<.05)$ y de manera negativa con la Escala de Autoeficacia Generalizada ( $r$ $=-.21 ; p<.05)$. El factor presente hedonista correlacionó positivamente con la Escala de Autoeficacia Generalizada $(r=.31 ; p<.05)$. Por último, el factor presente fatalista correlacionó positivamente con la Escala de Locus de Control $(r=.45 ; p<.05)$ y con la Escala de Malestar Psicológico $(r=.23 ; p<.05)$. En la Tabla 1 se pueden ver los resultados.

Tabla 1. Validez externa: Correlaciones del Inventario de Perspectiva Temporal de Zimbardo.

\begin{tabular}{cccccc}
\hline Escala & $\begin{array}{c}\text { Presente } \\
\text { hedonista }\end{array}$ & $\begin{array}{c}\text { Presente } \\
\text { fatalista }\end{array}$ & $\begin{array}{c}\text { Pasado } \\
\text { negativo }\end{array}$ & Futuro & $\begin{array}{c}\text { Pasado } \\
\text { positivo }\end{array}$ \\
\hline Locus de control & & .45 & .31 & \\
Malestar psicológico & & .23 & .43 & \\
Autoeficacia generalizada & .31 & & -.21 & \\
\hline
\end{tabular}


El estudio de la estabilidad de las puntuaciones del ZTPI se realizó a través del método de test-retest, se hizo con 30 casos y con un intervalo de un mes entre la primera y la segunda toma. Casi todos los resultados fueron estadísticamente significativos, variando entre .57 y .77 . Los factores de pasado negativo y presente fatalista demostraron tener los mayores coeficientes de correlación $(r=.77$; $p<.05)$, seguido por pasado positivo $(r=.67$; $p<.05)$, presente hedonista $(r=.61 ; p<.05)$ y por último futuro $(r=.57 ; p<.05)$.

Finalmente, se realizaron análisis para evaluar si existían diferencias en función del sexo y nivel educativo en la muestra. Con la finalidad de analizar la influencia del sexo para cada uno de los factores del ZTPI, se aplicó el Test de Diferencias de Medias para muestras independientes. Se constataron diferencias significativas en función del sexo en 4 de los 5 factores de la prueba, siendo las medias mayores en las mujeres que en los hombres. En el factor presente hedonista no se hallaron diferencias significativas. En la Tabla 2 se informan las medias y desviaciones estándar para las puntuaciones totales según sexo.

Tabla 2. Medias y desviaciones estándar para las puntuaciones totales del ZTPI según sexo.

\begin{tabular}{cccccccccccccccccc}
\hline Sexo & \multicolumn{3}{c}{ Pasado negativo } & \multicolumn{3}{c}{ Presente hedonista } & \multicolumn{3}{c}{ Futuro } & \multicolumn{4}{c}{ Pasado positivo } & \multicolumn{2}{c}{ Presente fatalista } \\
\hline & $\mathrm{n}$ & $\mathrm{M}$ & $\mathrm{DE}$ & $\mathrm{N}$ & $\mathrm{M}$ & $\mathrm{DE}$ & $\mathrm{n}$ & $M$ & $D E$ & $n$ & $M$ & $D E$ & $n$ & $M$ & $D E$ \\
Femenino & 168 & 2.68 & 0.76 & 180 & 3.47 & 0.55 & 175 & 3.65 & 0.48 & 177 & 3.85 & 0.5 & 173 & 2.46 & 0.6 \\
Masculino & 140 & 2.48 & 0.64 & 134 & 3.48 & 0.53 & 138 & 3.51 & 0.5 & 140 & 3.67 & 0.51 & 140 & 2.28 & 0.58 \\
\hline
\end{tabular}

Para analizar el nivel educativo se trabajó con tres grupos. Se consideró como bajo nivel educativo el de aquellas personas que tenían hasta secundario incompleto, nivel educativo medio el de aquellas con secundario completo y nivel educativo alto el de las personas con estudios superiores. Así, se procedió a realizar un análisis ANOVA, que indicó diferencias significativas en cuatro de los cinco factores del ZTPI. En la Tabla 3 se reportan las medias y desviaciones estándar para las puntuaciones totales en función del nivel educativo. En los factores pasado negativo, presente hedonista y presente fatalista, las puntuaciones obtenidas fueron mayores en personas con bajo nivel de educación. En el factor pasado negativo, las personas con bajo nivel educativo $(n=52)$ presentaron una media de 2.89 $(D E=.68)$ y las de alto nivel educativo $(n=92)$ de $2.45(D E=.7)(F=6.42 ; p<.005)$. En el factor presente hedonista, las personas con bajo nivel educativo $(n=49)$ presentaron una media de $3.66(D E=.57)$ y las de alto nivel educativo $(n=95)$ de $3.25(D E=.7)$ $(F=12.59 ; p<.005)$. En el factor presente fatalista las personas con bajo nivel educativo $(n=50)$ presentaron una media de 2.78 $(D E=.68)$ y las de alto nivel educativo $(n=94)$ de $2.21(D E=.49)(F=16.07 ; p<.005)$. Por su parte, las personas con un nivel alto de educación presentaron puntuaciones más altas en el factor futuro en relación a las personas de baja educación. Las personas con alto nivel educativo $(n=96)$ presentaron una media de $3.72(D E=.47)$ y las de bajo nivel educativo $(n=46)$ de $3.57(D E=.43)(F=5.28$; $p=.006)$. No se hallaron diferencias significativas en el factor pasado positivo. 
Tabla 3. Medias y desviaciones estándar para las puntuaciones totales del ZTPI según nivel educativo.

\begin{tabular}{|c|c|c|c|c|c|c|c|c|c|c|c|c|c|c|c|}
\hline \multirow{2}{*}{$\begin{array}{c}\text { Nivel } \\
\text { educativo }\end{array}$} & \multicolumn{3}{|c|}{ Pasado negativo } & \multicolumn{3}{|c|}{$\begin{array}{l}\text { Presente } \\
\text { hedonista }\end{array}$} & \multicolumn{3}{|c|}{ Futuro } & \multicolumn{3}{|c|}{ Pasado positivo } & \multicolumn{3}{|c|}{$\begin{array}{l}\text { Presente } \\
\text { fatalista }\end{array}$} \\
\hline & $n$ & $M$ & $D E$ & $N$ & $M$ & $D E$ & $n$ & $M$ & $D E$ & $\mathrm{n}$ & M & $\mathrm{DE}$ & $\mathrm{n}$ & M & $\mathrm{DE}$ \\
\hline Bajo & 52 & 2.89 & .68 & 49 & 3.67 & .57 & 46 & 3.57 & .431 & 51 & 3.8 & .63 & 50 & 2.78 & .68 \\
\hline Medio & 164 & 2.57 & .714 & 170 & 3.54 & .53 & 171 & 3.52 & .51 & 170 & 3.8 & .42 & 169 & 2.36 & .59 \\
\hline Alto & 92 & 2.45 & .7 & 95 & 3.25 & .501 & 96 & 3.72 & .48 & 96 & 3.71 & .58 & 94 & 2.21 & .49 \\
\hline
\end{tabular}

\section{Discusión}

Los objetivos de este estudio fueron conocer si la adaptación española del ZTPI es apropiada para su uso en Argentina y brindar información acerca de sus propiedades psicométricas en Buenos Aires.

En cuanto al primero, se pudo constatar que muchos de los ítems de la versión española eran apropiados en nuestro medio, pero 34 ítems debieron ser modificados para ser comprendidos correctamente. Esto llevó a realizar una versión argentina que incluyese las correcciones devenidas del estudio de jueces y de la respuesta de individuos argentinos a la versión española.

Además, este trabajo mostró evidencias de validez estructural de los cinco factores que se replicaron, también en todas las adaptaciones que se hicieron con anterioridad (Apostolidis et al., 2006; B. Reuschenbach en comunicación personal con Kolesovs el 24 de mayo de 2006; Díaz-Morales, 2006; Kolesovs, 2009; Milfont et al., 2008; Sircova et al., 2014). Esto daría cuenta de la universalidad de los constructos evaluados por el ZTPI, ya que los mismos factores se han encontrado empíricamente en diferentes culturas y países (Sircova et al., 2014).

El test-retest evalúa la varianza de error al paso del tiempo. Que las correlaciones de la evaluación test-retest hayan sido altas y positivas significa que las puntuaciones acerca de la orientación temporal, tal como son evaluadas en el ZTPI, presentan estabilidad a lo largo del tiempo. Aun de manera indirecta, estos resultados son compatibles con la idea de que estas orientaciones temporales son características relativamente estables de la personalidad en distintas etapas vitales.

En términos de validez de contenido, pudo notarse que la estructura de la evaluación de los expertos se correspondía con el análisis factorial de los autores del ZTPI (Zimbardo y Boyd, 1999), en donde los factores que lograron una mayor carga fueron los que tenían la mayor cantidad de acuerdos acerca de alta relevancia, coherencia y claridad. Aquellos factores que se mostraron como más definidos en el análisis factorial de Zimbardo y Boyd (1999) y como los más relevantes, coherentes y claros son el pasado negativo $\mathrm{y}$ presente hedonista, mientras que el menos definido por el factorial de los autores, y el menos relevante, coherente y claro fue el presente fatalista.

El pasado negativo y el presente hedonista se mostraron como los más definidos en el análisis factorial de Zimbardo y Boyd (1999) y como los más relevantes, coherentes y claros, mientras que el presente fatalista fue el menos definido y el menos relevante, coherente y claro.

También se observó que aquellos ítems que los evaluadores marcaron como relevantes, coherentes y claros eran también los que tenían mayor carga en el análisis factorial que se realizó en este trabajo. Algunos ejemplos son el ítem 8 del factor presente hedonista que tuvo una carga de .51 en el análisis factorial, el ítem 7 del factor pasado positivo que tuvo una carga de .55 , el ítem 10 del factor futuro 
que cargó con un valor de $.5 \mathrm{y}$, por último, el ítem 34 del factor pasado negativo que cargó con un valor de .68 .

En términos de validez externa, la relación entre presente hedonista y la escala de autoeficacia generalizada podría entenderse en términos de asertividad, es decir que la persona se encuentra capaz de expresar y seguir sus convicciones. La relación del presente fatalista con la escala de malestar psicológico podría estar sostenida desde la concepción de un hecho traumático pasado o experiencias negativas pasadas, que se perciben en el presente y que son fuente de malestar. Esto, a su vez, podría estar aparejado con un estilo atribucional externo, explicando también la correlación del factor presente fatalista con la escala de locus de control. Estas mismas conjeturas podrían usarse también para explicar las correlaciones del factor pasado negativo con la escala de malestar psicológico y la escala de locus de control. La correlación negativa del factor pasado negativo con la escala de autoeficacia generalizada podría entenderse como una falta de percepción de minusvalía y de falta de recursos para poder expresar y seguir sus convicciones.

Sircova et al. (2014) mencionan que las orientaciones de pasado negativo y presente fatalista aparecen, repetidas veces, como asociadas negativamente con bienestar psicológico y comportamientos respectivos a ese bienestar. Esto se ve también en una conclusión a la que arriban Oyanadel y Buela-Casal (2010) al mencionar que el pasado negativo se relaciona negativamente con la salud. Sircova et al. (2014) también señalan que las orientaciones de pasado positivo y futuro se encuentran, en la mayoría de casos, asociados con bienestar psicológico, mientras que el presente hedonista se encuentra simultáneamente relacionado a comportamientos de riesgo y comportamientos satisfactorios asociados a bienestar psicológico. Un ejemplo de lo descripto por Sircova et al. (2014) y Oyanadel y Buela-Casal (2010) es lo que muestran Astrom, Wiberg, Sircova, Wiberg y Carelli (2014) con respecto a pacientes ansiosos, indicando que en ellos existe un sesgo temporal hacia los aspectos negativos del pasado y del futuro, pero no del presente, lo cual aporta algo de conocimiento a la relación que existe entre orientación temporal y psicopatología.

Continuando con la relación entre orientación temporal y psicopatología, Oyanadel, Buela-Casal, Araya, Olivares y Vega (2014) ponen a prueba la posibilidad de modificar la orientación temporal y logran hacerlo con éxito. Estos hallazgos resultan relevantes, ya que demuestran que la orientación temporal podría incluirse como una variable terapéutica a la hora de diseñar intervenciones, cuyo fin sería fomentar la orientación temporal balanceada que se encuentra asociada positivamente a la salud (Oyanadel y Buela-Casal, 2010).

El presente trabajo representa una primera aproximación a la adaptación del Inventario de Perspectiva Temporal de Zimbardo a la Argentina. Las propiedades psicométricas que se estudiaron fueron la fiabilidad y validez estructural, la validez de contenido y la estabilidad de las puntuaciones. Los puntajes obtenidos en tales mediciones han sido satisfactorios, cumpliendo así con el objetivo general del trabajo.

En relación con los ítems utilizados para evaluar cada factor, siguiendo los resultados obtenidos tras la rotación varimax, es necesario mencionar algunas consideraciones. En primer lugar, los ítems 46, 24 y 56 poseen carga significativa positiva en el factor que es donde, siguiendo la prueba original, deberían cargar, pero a su vez, poseen una carga significativa negativa en otro factor que posee cualidad contraria, por lo tanto, estos ítems son consistentes porque la cualidad se está manteniendo. Similarmente, el ítem 1 debería cargar en el presente hedonista, pero tiene carga significativa en el pasado positivo; esto indica que se mantiene la cualidad de lo que se está evaluando, aunque en esta adaptación este ítem es más prístino para el pasado 
positivo. Por su parte, el ítem 39 tiene carga compartida entre el presente fatalista, donde debería cargar, y el pasado negativo. Ambos factores tienen la misma cualidad, por lo que se mantiene la medición, pero es probable que la redacción en negativo haya coadyuvado a la presencia de cargas superiores a .40 en ambos factores.

Seis de los quince ítems correspondientes al factor presente hedonista deberían ser eliminados de la escala, ya que o tienen carga compartida o cargan en un factor contradictorio al que deberían cargar. Por otro lado, los ítems 3 y 52, que evalúan la orientación presente fatalista, también deberían ser eliminados, ya que cargan en un factor contradictorio al que deberían hacerlo. De la misma manera, el ítem 6 correspondiente al factor futuro, también debería ser revisado, ya que carga en un factor contradictorio al que debería cargar. Por último, el ítem 5 que pertenece al factor pasado negativo, también debería ser eliminado porque posee una carga significativamente mayor en un factor cualitativamente contradictorio al que debería cargar. Estos señalamientos podrían ser tenidos en cuenta para generar una versión breve de la técnica.

Tomando en consideración la variabilidad en las puntuaciones del ZTPI, los resultados obtenidos en este estudio sugieren que la misma se debe, en parte, al nivel educativo y secundariamente al sexo. Respecto a la edad, un estudio previo reporta diferencias significativas para las dimensiones presente hedonista y futuro. Conforme aumenta la edad, el patrón de orientación al presente hedonista decrece. Lo contrario sucede con el predominio de la orientación futura. Esto implica que la orientación temporal de las personas tiende a moverse evolutivamente desde una orientación centrada en el presente hacia el futuro (Brenlla, Willis y Germano, 2016; Laureiro-Martínez, Trujillo y Unda, 2017). En relación a las diferencias halladas respecto del sexo, los resultados obtenidos en este estudio coinciden con los hallados por Oyanandel et al. (2014), en cuanto a una tendencia mayor en las mujeres a puntuar más alto en pasado positivo y con Salmerón Pérez, Gutiérrez-Braojos y Rodríguez Fernández (2017) respecto de la similitud en la perspectiva de futuro entre hombres y mujeres. Sin embargo, estas tres variables, edad, nivel educativo y sexo pueden explicar cierta variabilidad en las puntuaciones, pero no completamente. Esto implica que la orientación temporal puede ser tenida en cuenta como un rasgo de personalidad y las diferencias que las personas obtienen en las puntuaciones de esta escala se explican mayoritariamente debido a diferencias individuales.

Este trabajo cuenta también con algunas limitaciones. La primera de ellas es que la muestra se reduce a personas de la Ciudad Autónoma de Buenos Aires y del Gran Buenos Aires. Para realizar la adaptación definitiva del Inventario de Perspectiva Temporal de Zimbardo a la Argentina sería recomendable incluir en la muestra personas de otras regiones del país. Otra limitación es el tamaño de la muestra con la que se realizó el test-retest, viéndose afectadas las propiedades psicométricas de la estabilidad en el tiempo de los factores. Sería recomendable también trabajar con una muestra más grande para evaluar esta propiedad en la adaptación definitiva del ZTPI. También debe considerarse una limitación el hecho de que la estructura obtenida por el análisis factorial exploratorio debería ser validada con un estudio confirmatorio.

Por último, algunas futuras líneas de investigación, que resultaría interesante trabajar, serían la realización de la adaptación definitiva, la configuración de una versión más corta del ZTPI y comenzar a evaluar a pacientes clínicos para poder empezar a comprender acerca de los mecanismos temporales que operan en las psicopatologías y, en un futuro, utilizar la orientación temporal como variable para el diagnóstico y tratamiento de enfermedades psiquiátricas. 


\section{Referencias bibliográficas}

Apostolidis, T., Fieulaine, N., Simonin, L. y Rolland, G. (2006). Cannabis use, time perspective and risk perception: Evidence of a moderating effect. Psychology and Health, 21(5), 571-572. doi: 10.1080/14768320500422683

Astrom, E., Wiberg, B., Sircova, A., Wiberg, M. y Carelli M.G. (2014). Insights into features of anxiety through multiple aspects of psychological time. Journal of Integrative Psychology and Therapeutics, 2(1), 3. doi: 10.7243/20544723-2-3

Bordignon, N.A. (2005). El desarrollo psicosocial de Eric Erikson. El diagrama epigenético del adulto. Revista Lasallista de Investigación, 2(2), 50-63. Recuperado de http://www. redalyc.org/articulo.oa?id=69520210

Brenlla, M.E., Vázquez, N. y Aranguren, M. (2008). Adaptación Argentina de la Escala de Locus de Control de Rotter. Manuscrito no publicado.

Brenlla, M.E. y Aranguren, M. (2010). Adaptación argentina de la escala de malestar psicológico de Kessler (K10) Revista de Psicología de la Pontificia Universidad Católica del Perú, 28(2), 311-342. Recuperado de http://bibliotecadigital.uca.edu.ar/repositorio/ investigacion/ adaptacion-argentina-escala-malestar-kessler. pdf

Brenlla, M.E., Willis, B., y Germano, G. (2016). Estimación del tiempo y perspectiva temporal en distintas etapas de la adultez. Investigaciones en Psicología, 21(1), 27-34. Recuperado de http://www.psi.uba.ar/investigaciones/ revistas

Brenlla, M.E., Aranguren, M., Rossaro, M.F. y Vázquez, N. (2010). Adaptación para Buenos Aires de la Escala de Autoeficacia General. Interdisciplinaria, 27(1), 77-94.

Brenlla. M.E., Serna, J., Londra, F., Rodríguez, M.E. y Zapater, J. (2012) Adaptación Argentina del Inventario de Perspectiva Temporal de Zimbardo. Memorias del IV Congreso Internacional de Investigación y Práctica Profesional en Psicología. XIX Jornadas de Investigación. VIII Encuentro de Investigadores en
Psicología del Mercosur, Argentina, Tomo 2, 126. Recuperado de https://www.aacademica. org/000-072/984

Corral-Verdugo, V., Fraijo-Sing, B., y Pinheiro, J.Q. (2006). Sustainable behavior and time perspective: Present, past, and future orientations and their relationship with water conservation behavior. Interamerican Journal of Psychology, 40(2), 139-147. Recuperado de http:// www.redalyc.org/articulo.oa?id=28440201

Díaz-Morales, J.F. (2006). Estructura factorial y fiabilidad del Inventario de perspectiva temporal de Zimbardo. Psicothema, 18(6), 565-571.

Escobar-Pérez, J. y Cuervo-Martínez, A. (2008). Validez de contenido y juicio de expertos: una aproximación a su utilización. Avances en Medición, 6(1), 27-36.

Fraisse, P. (1984). Perception and estimation of time. Annual Review of Psychology, 35(1), 1-37. doi: 10.1146/annurev.ps.35.020184.000245

Galarraga, M.L., y Stover, J.B. (2016). Inventario de Perspectiva Temporal de Zimbardo: Adaptación en estudiantes de nivel medio de Buenos Aires. Psicodebate, 16(1), 109-128. doi: 10.18682/pd.v16i1.540

Holman, E.A. y Silver, R.C. (1998). Getting stuck in the past: temporal orientation and coping trauma. Journal of Personality and Social Psychology, 74(5), 146-163. Recuperado de https://webfiles.uci.edu/rsilver

James, W. (1945). Principios de psicología. Argentina: Glem. Trabajo original publicado en 1890.

Janeiro, I.N. (2012). O Inventário de Perspectiva Temporal: estudo de validação. Revista Iberoamericana de Diagnóstico y Evaluación Psicológica, 34(1), 117-133. Recuperado de http://www.aidep.org/03_ridep/R34/ ART\%206.pdf

Keough, K.A., Zimbardo, P.G. y Boyd, J.N. (1999). Who's smoking, drinking and using drugs? Time perspective as a predictor of substance use. Basic and Applied Social Psychology, 21(2), 149-164. doi: 10.1207/ S15324834BA210207

Kessler, R., y Mroczek, D. (1994). Final versions of our non-specific psychological distress 
scale. Memo dated March, 10, 1994. Ann Arbor (MI): Survey Research Center of the Institute for Social Research, University of Michigan. Recuperado de http://journals.sagepub.com/ doi/pdf/10.3109/00048674.2010.543653

Kolesovs, A. (2009). Factorial validity of the Latvian and Russian versions of the ZTPI in Latvia. Baltic Journal of Psychology, 10(1), 46-54. Recuperado de https://www.lu.lv/fileadmin/

Laureiro-Martinez, D., Trujillo, C.A., y Unda, J. (2017). Time Perspective and Age: A Review of Age Associated Differences. Frontiers in Psychology, 8, 101. doi: 10.3389/ fpsyg.2017.00101

Lewin, K. (1978). La teoría del campo en la ciencia social. Buenos Aires, Argentina: Paidós. Trabajo original publicado en 1951.

Marín, G. (1986). Consideraciones metodológicas básicas para conducir investigaciones psicológicas en América Latina. Acta Psiquiátrica y Psicológica de América Latina, 32(3), 183-92. Recuperado de https://dialnet.unirioja.es/ servlet $/$ articulo? codigo $=279456$

Martínez-Arias, R. (1995). Psicometría: Teoría de los test psicológicos y educativos. Madrid: Síntesis.

Milfont, T.L., Andrade, P.R., Belo, R.P. y Pesoa, V.S. (2008). Testing Zimbardo Time Perspective Inventory in a Brazilian sample. Interamerican Journal of Psychology, 42(1), 1-10. Recuperado de http://www.redalyc.org/articulo.oa?id=28442106

Nuttin, J. (1964). The future time perspective in human motivation and learning. Acta Psychologica, 23(1), 60-82. doi: 10.1016/00016918(64)90075-7

Nuttin, J. (1985). Future time perspective and motivation: theory and research method. Estados Unidos: Erlbaum.

Nuttin, J. y Lens, W. (1980) Motivation et perspective d'avenir. Louvain: Presses Universitaires de Louvain

Oyanadel, C. y Buela-Casal, G. (2010). La percepción del tiempo: influencias en la salud física y mental. Universitas Psychologica, 10(1), 149-161. Recuperado de https://search. proquest.com/openview/0e334c7bc17fc- 4610f616006bf5abc4a/1?pq-origsite=gscholarycbl=2041157

Oyanadel, C., Buela-Casal, G., y Pérez-Fortis, A. (2014). Propiedades psicométricas del inventario de orientación temporal de Zimbardo en una muestra chilena. Terapia Psicológica, 32(1), 47-55. doi: 10.4067/S071848082014000100005

Oyanadel, C., Buela-Casal, G., Araya, T., Olivares, C. y Vega, H. (2014). Percepción del tiempo: resultados de una intervención grupal breve para el cambio del perfil temporal. Suma Psicológica, 1(21), 1-7. doi: 10.1016/S01214381(14)70001-3

Piaget, J. (1970). The child's conception of time. Estados Unidos: Basic Books. Trabajo original publicado en 1969.

Poortinga, Y.H. (1989). Equivalence of Cross Cultural data: an overview of basic issues. International Journal of Psychology, 24(6), 737-756. doi: 10.1080/00207598908247842

Roeckelein, J.E. (2000). The concept of time in psychology: A resource book and annotated bibliography. California, Estados Unidos: Greenwood Publishing Group.

Rotter, J.B. (1966). Generalized expectancies for internal versus external control of reinforcement. Psychological Monographs, 80(1), 1-28. doi: 10.1037/h0092976

Salmerón Pérez, H., Gutiérrez-Braojos, C., y Rodríguez Fernández, S. (2017). The relationship of gender, time orientation, and achieving self-regulated learning. Revista de Investigación Educativa, 35(2), 353-369. doi: 10.6018/rie.35.2.273141

Schwarzer, R. y Jerusalem, M. (1995). Generalized Self-Efficacy Scale. En J. Weinman, S. Wright y M. Johnston (Eds.), Measures in health psychology: A user's portfolio. Causal and control beliefs (pp. 35-37). Windsor, United Kingdom: Nfer-Nelson.

Sircova, A., van de Vivjer, F.J.R., Osin, E., Milfont, T.L., Feulaine, N., Kislali-Erginbilgic, A., Zimbardo, P.G. y 54 miembros del International Time Perspective Research Project (2014). A Global Look at Time: A 24-Country Study of the Equivalence of the Zimbardo 
Time Perspective Inventory. SAGE Open, 4(54), 1-12. doi: 10.1177/2158244013515686

Skinner, B.F. (1985). Aprendizaje y comportamiento. Barcelona, España: Martínez Roca.

Strathman, A., Gleicher, F., Boninger, D. y Edwards, C. (1994). The consideration of future consequences: Weighing immediate and distant outcomes of behavior. Journal of Personality and Social Psychology, 66(4), 742-752. doi: 10.1037/0022-3514.66.4.742

Vásquez Echeverría, A., Martín, A.E.C., Ortuño, V., Esteves, C., y Joireman, J. (2017). Adaptación y validación inicial al castellano de la Escala de Consideración de las Consecuencias Futuras. Revista Iberoamericana de Diagnóstico y Evaluación Psicológica, 44(2), 5-15. doi: 10.21865/RIDEP44.2.01

Wessman, A.E. (1973). Personality and the subjective experience of time. Journal of
Personality Assessment, 37(2), 103-114. doi: 10.1080/00223891.1973.10119839

Zaleski, Z. (1996). Future anxiety: Concept measurement and preliminary research. Personality and Individual Differences, 21(2), 165-174. doi: 10.1016/0191-8869(96)00070-0

Zimbardo, P.G.; Haney, C.; Banks, W.C. y Jaffe, D. (1973, 8 de abril). The mind is a formidable jailer: A Pirandellian prision. New York Times Magazine, p.36. Recuperado de http://www. nytimes.com/1973/04/08/

Zimbardo, P.G. y Boyd, N. (1999). Putting time in perspective: a valid, reliable, individual-differences metric. Journal of Personality and Social Psychology, 17(6), 1271-1288. doi: 10.1037/0022-3514.77.6.1271

Zimbardo, P.G. y Boyd, N. (2008). The time paradox: The new psychology of time that will change your life. Estados Unidos: Atria Books.

Recibido: 22 de noviembre de 2017 Aceptado: 18 de septiembre de 2019 
Brenlla, Zapater y Germano 